To the Editors:

\title{
Authors' reply re: HLA-B27 allele frequency in Sri Lankan patients with spondyloarthritides
}

\author{
S Kidnapillai' ${ }^{1}$, D D Sirisena', V H W Dissanayake ${ }^{1,2}$
}

Ceylon Medical Journal 2016; 61: 203

10.4038/cmj.v61i4.8399

The HLA-B27 genotype detection described in this article was carried out at the only centre providing HLA-B27 genotyping in the country. Patients from all parts of the country are referred to this centre for HLA-B27 genotyping.

The age distribution of the 373 spondyloarthritides (SA) patients ranged from 6 to 77 years, comprising of 249 males and 124 females. The patients in this cohort were referred by the physicians for HLA-B27 testing, we do not have the data on the percentage distribution of the exact type of SA in these patients, as this information was not included in most of the referral letters.

The authors agree with the reader's comment that if this cohort of SA patients had only a few AS patients, it would reduce the overall frequency of the HLA-B27 alleles. This point has been addressed in the published paper where we mentioned that in contrast to published literature from other populations, the relatively lower allele frequency $(22.8 \%)$ obtained in this study could probably be attributed to the small sample size.

To the authors' knowledge, this is the first study that was carried out to determine the frequency of HLA-B27 allele in a cohort of Sri Lankan patients with SA referred from all parts of the country to the only diagnostic centre providing HLA-B27 genotyping services during the study period. We agree that this cohort does not represent all the Sri Lankan patients with SA, however given the fact that there have been no previously published data on the frequency of HLA-B27 allele in Sri Lankan patients with SA, the findings of this study serve to provide preliminary data on the existence of the HLA-B27 allele in a sample of Sri Lankan patients with SA.

The authors agree with the reader's comment that only a select group of SA patients would enter the database for HLA-B27 testing as this is a paid test carried out in a private hospital. However, we do not think that the title of this article is misleading because we have clearly stated that the main aim of this study was to assess the frequency of this allele in Sri Lankan patients referred for HLA-B27 testing using an anonymized database of HLA-B27 test results of 373 Sri Lankan patients with SA referred from all parts of the country.

We agree that if a sizeable sample of consecutive patients with SA in a busy rheumatology clinic in a state hospital is studied, a more accurate picture of the HLA-B27 allele frequency could be obtained that can be construed as representative of Sri Lankan patients with SA.

${ }^{1}$ Human Genetics Unit, Faculty of Medicine, University of Colombo, ${ }^{2}$ Asiri Centre for Genomic and Regenerative Medicine, Asiri Surgical Hospital, Colombo, Sri Lanka.

Correspondence: VHWD, e-mail:<vajirahwd@hotmail.com>. 